in colorectal cancer cells by decreasing the sensitivity of DLD1 cells to combination anticancer drugs used in clinics for the treatment of colorectal cancer, oxaliplatin and 5-fluouracil. Analysis of accumulation of senescenceassociated beta-galactosidase showed that neither DLD1, nor DLD1-Wip1ON cells don not develop senescent phenotype and their resistance is not based on induction of senescence. To prove that resistance of DLD1-Wip1ON cells occur due to Wip1 overexpression, we created DLD1-Wip1KO cell line with bi-allellic knockout of Wip1 gene. The opposite effect was observed in cells with deletion of Wip1 making them more sensitive to combination of DNA damaging drugs. Conclusions: Overexpression and deletion of nuclear phosphatase Wip1 affects DNA damage response in colorectal cancer cells induced by a classical combination of chemotherapeutic drugs used for FOLFOX adjuvant therapy of colorectal cancer.

The reported study was funded by RFBR according to the research project № 17-04-01592.

doi: http://dx.doi.org/10.7124/bc.0009E2

\section{G-4. A novel approach in studies of the posttranslational modification effect on YB-1 translocation}

\section{E. M. Grigorieva, D. A. Mordovkina,}

E. R. Kim, L. P. Ovchinnikov

Institute of Protein Research, Russian Academy of Sciences, Pushchino, Russia

kategrigoreva@vega.protres.ru
The Y-box binding protein 1 (YB-1) is a multifunctional protein. In the cytoplasm, it is involved in mRNA translation and stabilization, while in the nucleus it participates in replication regulation and DNA transcription. Mostly, YB-1 shows the cytoplasmic localization, but in some cases (under stress or modification) it goes to the nucleus. Its best studied nuclear translocation-causing modification is phosphorylation at S102. The in vitro transport assay technique is a simple and convenient method to study the mechanisms of protein transport. Its advantage is the possibility to control transport conditions. Phosphorylation is a posttranslational modification that regulates many cellular events including protein translocation. To study its effect, a phosphomimetic technique is used, in which amino acid substitutions are introduced into phosphorylation sites to simulate the dephosphorylated state (replacement by Ala) and the phosphorylated state (by Asp or Glu). We used a combination of these two techniques to find out the effect of YB-1 modifications on its nucleus-cytoplasm transport. Methods The sitedirected mutagenesis was used to make mutations in the YB-1 phosphorylation sites. The proteins were isolated and the transport assay system was assembled according to [1,2]. For the import reaction, we used YB-1 with S102 replaced by alanine or aspartic acid. Protein localization was assessed by immunofluorescence microscopy. Results In the transport assay system, two types of cell culture conditions were used: standard conditions (YB-1 in the cytoplasm) and transition-stimulating conditions (YB-1 in the nucleus). If phosphorylation stimulates the transition, the phosphomimetic mutant will show the nuclear localization even in standard conditions, while the dephosphory- 
lated one will be detected in the cytoplasm under transition-stimulating conditions. If phosphorylation inhibits the transition, the phosphomimetic mutant will be detected in the cytoplasm in stimulating conditions, while the dephosphorylated one will localize to the nucleus under standard conditions. As expected, YB-1 S102A was absent from the nucleus under transition-stimulating conditions, while YB-1 S102D was detected there in standard conditions. Conclusions Thus, phosphorylation at S102 activates nuclear translocation of YB-1, which is in agreement with the literature data $[3,4,5]$. Hence, the proposed approach can be used in studies of the posttranslational modification effect on protein transport.

This work was supported by the Russian Foundation for Basic Research (\# 18-34-00359\18).

References 1. Evdokimova V. et al. The EMBO journal. 2001; 20; 5491-5502. 2. Mordovkina D. A. et al. BBRC. 2016; 480; 629-34. 3. Sutherland B. W. et al. Oncogene. 2005; 24; 4281. 4. Basaki Y. et al. Oncogene. 2007; 26; 2736. 5. Kosnopfel C. et al. Molecular Cancer Research. 2018; 16; 1149-60.

doi: http://dx.doi.org/10.7124/bc.0009E3

\section{H-1. Mg, K-containing microparticle: a possible active principle of $\mathrm{EM}$ fermentation product}

Toru Higashinakagawa ${ }^{1,2}$, Haruhisa Kikuchi $^{3}$ and Hidekazu Kuwayama ${ }^{4}$

${ }^{1}$ Tokyo Women's Medical University; ${ }^{2}$ EM Research Organization; ${ }^{3}$ Tohoku University; ${ }^{4}$ University of Tsukuba, Japan.

toru@waseda.jp
EM represents Effective Microorganisms, a microbial consortium consisting mainly of photosynthetic bacteria, lactic acid bacteria and yeast. Various effects on cells of EM fermentation product were reported in the previous WBW25 Meeting in 2017. Here, we report our attempt to identify its active principle and the ongoing preliminary results. The activity was assayed by promoted formation of fruiting body of Dictyostelium discoideum. The EM fermentation product was first subjected to liquid-liquid separation, with the activity being recovered in aqueous phase. Concentrated aqueous fraction was further subjected to column chromatography. No activity was detected in any eluant, while almost all activity was recovered in residual insoluble material. The application of conventional organic chemistry procedures did not lead to any informative results. Acid treatment of the insoluble material produced air bubbles, suggesting it to be composed of some inorganic carbonate. Viewed under scanning electronmicroscope, these residues revealed spherical particles of $\mu \mathrm{m}$ range. Energy Dispersive X-ray (EDX) Spectroscopy pointed to the existence of magnesium and, to a certain extent, potassium. In a separate experiment, acid treatment and alkali neutralization of EM fermentation product completely wiped out the stimulatory activity of fruiting body formation. These lines of evidence indicate these $\mathrm{Mg}, \mathrm{K}$-containing microparticle to be active principle of EM fermentation product, at least, in regard to fruiting body formation in Dictyostelium discoideum. How these particles exert their effect is currently under extensive investigation. 